\title{
Las fronteras de la pobreza en Bogotá
}

\section{Consuelo Uribe Mallarino, ${ }^{*}$ Jefferson Jaramillo Marín**}

Perfiles Latinoamericanos, $24(48)$

2016 | pp. 243-264

DOI: $10.18504 / \mathrm{pl} 2448-010-2016$

\begin{abstract}
Resumen
Este artículo propone una perspectiva de análisis sobre la pobreza con un enfoque multidimensional, que la considera no solo como una suma de variables e indicadores, sino que le agrega una estrategia exploratoria sobre cómo se percibe la pobreza entre grupos que reciben o no programas para aliviarla, o usan o no servicios públicos subsidiados. Con base en herramientas cuantitativas y cualitativas aplicadas a información recabada sobre una muestra de residentes de Bogotá, se estudian las maneras en que las fronteras de sentido sobre la pobreza se relacionan con factores objetivos y subjetivos, lo cual implica ser o no usuarios de programas de subsidios, tener una condición socioeconómica y educativa determinada, además de las características de participación política, grado de asociación y uso de mecanismos para hacer valer derechos ciudadanos.
\end{abstract}

\section{Abstract}

The article proposes an analytical perspective on poverty with a multidimensional approach, not only as an aggregate of variables and indicators, but also using a probing strategy that considers the way in which it is perceived between the groups receiving programs to alleviate poverty or use subsidized public services, and those without. Based on tools for quantitative and qualitative analysis applied to data collected on a sample of residents of Bogota, the authors analyze how the boundaries of sense on poverty are related to both objective and subjective factors related to being or not users of these programs, socioeconomic and educational conditions, as well as political participation, degree of association and use of mechanisms to enforce civil rights.

Palabras clave: pobreza multidimensional, fronteras de la pobreza, pobreza subjetiva, representaciones sociales, estratificación social, Bogotá.

Keywords: Multidimensional poverty, poverty frontiers, subjective poverty, social representations, social stratification, Bogota.

* Doctora en Antropología Social por la Ecole des Hautes Etudes en Sciences Sociales (eHess), Francia. Profesora Titular y, hasta marzo de 2016, vicerrectora de Investigación de la Pontificia Universidad Javeriana, Bogotá, Colombia.

* Doctor de Investigación en Ciencias Sociales por la Flacso, México. Profesor Asociado y director del Departamento de Sociología de la Pontificia Universidad Javeriana, Bogotá, Colombia. 


\section{Introducción ${ }^{1}$}

L

a pobreza es objeto de análisis de investigadores y consultores en todo el mundo. Alrededor de ella se construyen índices, líneas, mapas, coeficientes y encuestas de todo tipo. De ella se ocupan diversas estrategias de intervención, labor en la que participan gobiernos, organizaciones filantrópicas y humanitarias, agencias de cooperación y de desarrollo. En los Objetivos de Desarrollo del Milenio, la "superación" de la pobreza ha sido el primero de los ocho de la lista, mostrando la prioridad que merece el asunto. Luego de varias décadas de estudio y gestión institucional de la problemática, no existe un consenso sobre su definición o un modelo único de intervención, lo que hace de la pobreza un campo de disputas y tensiones. ${ }^{2}$

En medio de este campo tan impugnado y debatido, son cada vez más comunes las aproximaciones analíticas y empíricas de carácter multidimensional que buscan trascender los enfoques unidimensionales de definición de la pobreza como carencia de ingresos y de su intervención por la vía exclusiva de las transferencias. En esta perspectiva multidimensional se sitúa este artículo, resultado de un proyecto de investigación realizado en la ciudad de Bogotá, entre 2008 y 2010. Su principal objetivo es comprender, desde la perspectiva de los ciudadanos, las "fronteras de sentido" sobre la pobreza en Bogotá. Estas fronteras o marcos de representación incluyen dimensiones subjetivas, culturales, económicas y políticas, que no logran ser percibidas en las habituales clasificaciones estadísticas por rangos de ingreso y por carencias de bienes, activos y servicios.

El principal aporte del artículo es identificar que el sentido de estas fronteras depende principalmente, aunque no solo de ello, de tres factores, a la vez objetivos y subjetivos, que están representados por las percepciones y valoraciones de cuatro grupos de individuos de los cuales hablaremos luego: a) dependencia de los ciudadanos respecto de los programas de protección estatal, b) aceptación por parte de estos ciudadanos de la protección institucional de programas y subsidios para aliviar la pobreza y de los servicios sociales públicos, y c) reconocimiento-cumplimiento, tanto de parte del Estado como de los ciudadanos, de

1 Este artículo se basa en el proyecto de investigación (ID 1998) "Las fronteras de la pobreza en Bogotá" que realizó el grupo Política Social y Desarrollo, entre febrero de 2008 y junio de 2010. Contó con financiación de la Oficina para el Fomento de la Investigación (OFI) de la Pontificia Universidad Javeriana (Colombia). Participaron Silvia Cogollos, Jaime Ramírez, Socorro Vásquez, Jefferson Jaramillo y Consuelo Uribe, y los egresados de Sociología, Tyanif Rico y de Historia, Diego Barbosa.

2 Para una ampliación de la pobreza como campo de disputas en términos de las estrategias de intervención y las prácticas y discursos alrededor de su definición, se recomienda Rojas (2004), Escobar (1996) y Spicker, Álvarez \& Gordon (2009). 
un conjunto de deberes y derechos que caracterizan a una sociedad de derecho. Estos factores fueron construidos a partir de la combinación y significancia estadística de una serie de variables activas resultado del análisis de una encuesta (véanse los anexos 1 y 2). El enfoque exploratorio adoptado permite identificar factores conceptuales latentes en datos que, potencialmente, pueden ayudar a ajustar los modelos teóricos relacionados con la problemática de estudio, en este caso, la pobreza multidimensional.

El artículo se divide en cuatro apartados. En el primero se discuten las especificidades de la problemática investigada y su ruta teórica. En el segundo se describen los presupuestos metodológicos tanto cualitativos como multivariados que se utilizaron, en específico la combinación de dos técnicas: el análisis factorial de correspondencias múltiples (ACM) y el análisis de clasificación o cluster analysis (CA). El tercer apartado realiza un breve recorrido contextual sobre la situación reciente de la pobreza en Colombia, concretamente en Bogotá. El cuarto apartado explica algunos de los resultados, la discusión analítica y las conclusiones centrales alrededor de cómo se trazan las fronteras de la pobreza en Bogotá. Por último, se presenta una reflexión concluyente. Los resultados se exponen de acuerdo a una clasificación de cuatro grupos homogéneos de ciudadanos, tomando como base su varianza frente a los tres factores arriba mencionados: beneficiarios confiados, empoderados, relativistas y escépticos descontentos.

\section{Problemática investigada, hipótesis y perspectivas teóricas}

El proyecto de investigación del cual deriva este artículo tuvo como propósito reconocer los aspectos subjetivos, económicos, sociales, culturales y políticos que conducen a que los habitantes de Bogotá tracen unas fronteras de sentido sobre qué es ser o no pobre. La categoría de frontera funciona en el análisis como un marco de representación ${ }^{3}$ utilizado por los ciudadanos para delinear, situar o percibir relativamente si viven o no en condición de pobreza en la ciudad y en los barrios en los que habitan, de acuerdo con varias dimensiones: si usan o no subsidios, si se autoadscriben en una u otra clase social y estrato socioeconómico, a situaciones particulares de vulnerabilidad y adversidad, y a la utilización de estrategias para afrontarlas, como la construcción de redes de solidaridad o la utilización de sus derechos ciudadanos o los mecanismos de

3 La noción de marco de representación se apoya en las perspectivas de Moscovici (2000), Jodelet (1984), Potter (1998), Vasilachis (2003), Jaramillo (2008) y Bayón (2012). En general, estos autores hablan de las representaciones como un conjunto de sistemas de interpretación que tienen las personas y que les permiten ordenar, clasificar, identificar, comunicar y nombrar los distintos aspectos de su vida. 
participación política. Tal marco de representación es influido a su vez por la clasificación que sobre estos ciudadanos realiza la política pública en términos de los subsidios y programas a los que son o no acreedores por su condición de población vulnerable o pobre. Las fronteras de la pobreza no solo corresponden a las de las personas que "objetivamente" viven en los barrios pobres, sino que incluyen "percepciones" de personas residentes en todos los estratos socioeconómicos. ${ }^{4}$ En Colombia, un estrato socioeconómico clasifica una vivienda urbana de acuerdo con criterios sociourbanísticos, a fin de que reciba un régimen diferencial de impuesto predial y de tarifas de servicios públicos domiciliarios. En Bogotá, como en las otras ciudades del país, la población se reparte en seis estratos, en una composición que no es proporcional en cada uno de ellos, lo que refleja la diversa capacidad de pago de los residentes. ${ }^{5}$

La idea de trabajar con la noción de frontera surgió de una investigación realizada en Bogotá en 2006, con la que se identificó la existencia de unas "fronteras flotantes" entre los residentes de distintos estratos socioeconómicos de la ciudad, las cuales sirven para que estos tracen un límite entre pobre y no pobre, de acuerdo con la posición que ocupan en la escala social (Uribe, Vásquez \& Pardo, 2006; Uribe, 2008). Este hallazgo preliminar condujo a que en esta oportunidad nos preguntáramos: a) ipueden estas fronteras indicar que un residente no solo ocupa un lugar particular en el espacio social de la ciudad debido a su condición material o socioeconómica, sino además revelar que su posición se caracteriza por producir y reproducir prácticas, construir experiencias subjetivas y representaciones sociales sobre su condición en ella?, b) ¿influyen las políticas públicas sobre la pobreza en la ubicación de estas fronteras y en las representaciones sobre el bienestar de los residentes? La hipótesis de partida fue que la identificación y clasificación de quién es pobre es altamente (aunque no únicamente) influenciada por las políticas públicas, sobre todo por las que definen quién es y quién no es beneficiario de programas estatales para aliviarla o superarla, y ello determina la manera de construirse el componente intersubjetivo de la pobreza.

4 En Colombia, las viviendas de los estratos uno, dos y tres reciben subsidios en forma decreciente para las tarifas de servicios públicos como acueducto, energía, aseo, teléfono fijo y gas domiciliario; aquellas del estrato cuatro pagan las tarifas de costo y las de los dos estratos superiores pagan sobrecostos a modo de contribución en un esquema de subsidios cruzados. Esta clasificación para garantizar cobros también tiene repercusiones socioculturales en las formas de percibir a las personas y ordenar la relación de ellas con el mundo social (Uribe, Vásquez \& Pardo, 2006). En sentido estricto, no son las personas las que están estratificadas, sino su residencia; pero en el imaginario colectivo, el rótulo del estrato se extiende a personas, instituciones educativas, centros comerciales, parques y varios tipos de prácticas (Uribe, 2008).

5 La población está repartida del siguiente modo: $9.4 \%$ en el estrato uno, $39.4 \%$ en el dos; $35.7 \%$ en el tres; $9.5 \%$ en el cuatro; $2.6 \%$ en el cinco; $1.7 \%$ en el seis y $1.6 \%$ sin estratificar. 
Este artículo reconoce la importancia de varias investigaciones en las que los componentes intersubjetivos de la pobreza han sido analizados, los cuales fundamentan también la necesidad de una indagación sobre las fronteras de la pobreza en Bogotá, como la que proponemos aquí. Por ejemplo, los trabajos de Liu (2008) y Roland-Levy et al. (2001) abordan la relación entre las condiciones de vida y las representaciones sociales y culturales acerca de ellas. Por su parte, el denominado enfoque de bienestar subjetivo ha mostrado cómo una indagación desde los mismos sujetos acerca de sus condiciones de vida históricas y sus autopercepciones sobre lo que es o no ser pobre, revela enormes divergencias respecto a los modelos de imputación/asignación de los expertos y desde la misma noción de carencia de ingresos. Esto lo destacan los estudios de Alkire (2007), Kakwani \& Silber (2008) y Rojas \& Jiménez (2008).

Los denominados enfoques multidimensionales de pobreza son centrales en esta dirección. Incorporando la concepción de Amartya Sen (1976) de que la pobreza se expresa en aspiraciones no cumplidas de los individuos, traducidas en múltiples privaciones, estos enfoques han contribuido con miradas participativas y autoperceptivas a complejizar las tradicionales concepciones sobre el tema basadas en la medición del ingreso, consumo de los hogares y líneas de pobreza que expresan la brecha de los ingresos necesarios para adquirir una canasta básica de alimentos. En esta dirección crítica destaca La voz de los pobres, estudio del Banco Mundial que buscó incidir en la reducción de invisibilidad de los pobres en el mundo señalando las potencialidades de una estrategia participativa y colaborativa de trabajo con ellos (Narayan, 2000).

En Colombia, el Departamento Administrativo Nacional de Estadística (DANE) y diferentes administraciones distritales de Bogotá han incorporado en los últimos años componentes intersubjetivos, mediante preguntas sobre si el hogar se considera o no pobre (Ravallion \& Lokshin, 1999: p. 32; Pinzón, 2014: p. 6). Estudios como los de Jiménez \& González (2014) muestran cómo desde 1997 se ha estado calculando un índice multidimensional de la pobreza (IMP) en el país, que descubre una situación paradójica: un importante descenso en la pobreza en varias regiones colombianas, a pesar de la fuerte inequidad social existente en varias de ellas, especialmente en las costas Caribe y Pacífica. ${ }^{6}$ En 2011, el gobierno colombiano, vía el Departamento Nacional de Planeación (DNP), acogió el índice multidimensional de pobreza propuesto por

6 Jiménez \& González muestran esta situación paradójica a partir del cálculo del IMP para ocho regiones del país. También muestran, sobre la base del coeficiente de Gini calculado a partir de los salarios, cómo Bogotá, respecto a tres ciudades capitales del país (Bucaramanga, Cali y Medellín), tiene un Gini de 0.53 , frente a los otros que están por debajo del 0.50 , lo que manifiesta la inequidad en la distribución del ingreso en esta ciudad, pese al descenso en la pobreza. Bonilla (2009: pp. 103-105), utilizando un método de microdescomposición paramétrica, encuentra que entre 2006 y 2007, los departamentos y 
Alkire y Foster desarrollado a su vez por la iniciativa en Pobreza y Desarrollo Humano de la Universidad de Oxford (OPHI). En la versión colombiana, este índice contempla cinco dimensiones: condiciones educativas del hogar, condiciones de la nińez y la juventud en el hogar, empleo, aseguramiento y acceso a servicios de salud, acceso a servicios públicos y condiciones de la vivienda, las cuales se ponderan para producir quince indicadores (Ángulo, Díaz, Pardo \& Riveros, 2011). ${ }^{7}$

Aunque los estudios sobre pobreza cada vez tienden a privilegiar los componentes intersubjetivos y los enfoques multidimensionales, es clave reconocer que la definición de pobreza prevaleciente y generalizada es la de privación de ingresos. El carácter unidimensional de la definición lleva, desde luego, a intervenciones de fácil operatividad, pero con enormes costos para las poblaciones por vía, única y exclusivamente, de transferencias de ingresos. Una muestra de esto último son los múltiples programas de transferencia de subsidios condicionados a la asistencia escolar o a controles de salud que se usan ampliamente como estrategia contra la pobreza en varios países de la región (Lampis, 2011; Ceccini \& Madariaga, 2011). Lo problemático aquí es que, aunque la intervención del Estado crece en forma de programas y subsidios para aliviar y combatir la pobreza, aspecto reconocido incluso por algunos grupos de población en Bogotá (como se verá más adelante), la investigación acerca de los efectos de estas intervenciones y políticas públicas en los individuos, así como en sus representaciones de su bienestar, brilla por su ausencia. En esta dirección creemos que este artículo puede aportar para profundizar en la discusión.

\section{Presupuestos metodológicos y modelo de análisis exploratorio}

En el análisis triangulamos información proveniente de dos estrategias metodológicas. La primera fue una encuesta realizada en 2008 a 960 personas residentes en diecisiete de las veinte localidades de Bogotá y de los seis estratos socioeconómicos de la ciudad. ${ }^{8}$ El $80 \%$ de las encuestas fueron aplicadas

las ciudades más ricos tienden a presentar peores indicadores de desigualdad, quizá determinados por factores educativos, demográficos y de composición de los hogares.

7 El imp propuesto por Alkire \& Foster (2009) se compone de las dimensiones de salud, educación, y nivel de vida, y diez indicadores: mortalidad infantil, nutrición, matrícula escolar, años de instrucción, bienes, piso del hogar, electricidad, agua, saneamiento, y combustible para cocinar. Cada dimensión resulta de la agregación de un cierto número de indicadores.

8 La muestra es representativa de la población residente en los distintos estratos por localidad. Se trata de una muestra probabilística y multietápica. Las localidades donde se aplicaron las encuestas fueron Barrios Unidos, Bosa, Chapinero, Ciudad Bolívar, Engativá, Fontibón, Kennedy, Los Mártires, Puente Aranda, Rafael Uribe, San Cristóbal, Santafé, Suba, Teusaquillo, Tunjuelito, Usaquén, 
en localidades de los estratos uno, dos y tres (las más pobladas y pobres de la ciudad) a personas de entre 18 y 55 años de edad o más, con una distribución homogénea de hombres y mujeres. De las personas de la muestra, el $47 \%$ nació en Bogotá y el 53\% fuera de la capital. De estos últimos, el 11\% llegó a Bogotá con sus padres desde que eran muy pequeños, el $24 \%$ lo hizo por razones de trabajo y $4.5 \%$ por violencia. El $66 \%$ de estos hogares, al momento de la encuesta, se componía de tres a cinco miembros. El 61\% de estos hogares tenían de uno a tres menores de edad. El 56\% de los residentes contaba con vivienda propia y el $42 \%$ con vivienda arrendada. El $27 \%$ tenía empleo formal, el $27 \%$ laboraba de forma independiente y el $11 \%$ se encontraba desempleado. El 26\% reconoció haber cursado hasta quinto de primaria, el 38\%, algún grado de educación media secundaria, y el 23\%, algún nivel universitario. Finalmente, el 56\% reconoció ganar entre cien y cuatrocientos mil pesos mensuales (aproximadamente de cincuenta a doscientos dólares).

La segunda estrategia metodológica (entrevistas y grupos focales) se aplicó a personas que recibían subsidios por estar catalogados como población vulnerable, o que no los recibían pero estaban ubicados en localidades clasificadas como pobres. ${ }^{9}$ Las dieciséis entrevistas realizadas entre los cinco grupos focales permitieron recabar percepciones y valoraciones sobre la condición de estas poblaciones en la ciudad, concretamente dentro de los barrios que habitan o habitaban, así como opiniones sobre los programas de subsidios estatales.

Para la interpretación de los datos de la encuesta se utilizó el análisis factorial de correspondencias múltiples (AFCM). Este método, conectado a la escuela francesa de análisis descriptivo multivariado (Lebart, Morineau \& Tabard, 1977) permite representar, en una perspectiva bidimensional, la dispersión y concentración de las observaciones obtenidas en una encuesta, para ubicarlas en un campo visual y analítico que divide el espacio en cuatro cuadrantes donde se sitúan los grupos conformados por la clusterización de factores representativos (gráficas 1 y 2). Con el AFCM se busca reducir las dimensionalidad de los datos, pero también la clasificación de individuos, ya que el método tiene la propiedad de transformar variables categóricas en cuantitativas. No obstante, es clave considerar que, desde el punto de vista de las escalas básicas de medida, el índice construido mediante este método representa una métrica diferente.

Usme. No se aplicó en La Candelaria, Sumapaz ni Antonio Nariño. El paquete estadístico utilizado fue el spss.

9 Los grupos focales se distribuyeron así: a) usuarios del programa Familias en Acción, b) funcionarios públicos de Acción Social y la Red Juntos, c) estudiantes de dos colegios públicos, d) madres cabeza de familia usuarias del Sistema de Identificación de Potenciales Beneficiarios de Programas Sociales (Sisbén 1). 
El AFCM es un método que "permite revelar las estructuras profundas a través de las variables manifiestas que proponen los registros estadísticos" (Gollac, 2009: p. 6; Bourdieu, 1984). En nuestro caso, este modelo se consideró como el más adecuado para representar las percepciones, o estructuras profundas de significado, de los cuatro grupos identificados alrededor de las fronteras de la pobreza, dentro de un espacio social figurado en un plano de cuadrantes.

El análisis de la encuesta se realizó combinando el análisis factorial de correspondencias múltiples con el análisis de conglomerados (cluster analysis). Con la primera técnica se identificaron estructuras subyacentes en todas las variables de la encuesta y se expresaron en nuevas variables cuantitativas (factores). Por esta vía se identificaron unas variables de perfil (ilustrativas) y unas variables temáticas (activas). Las ilustrativas correspondieron a estrato socioeconómico, sexo, edad, rol en el hogar, tamaño del hogar, tipo de vivienda, tenencia de vivienda, ocupación, nivel educativo, menores de 14 años, gasto en alimentos y origen de nacimiento. Mientras que las variables activas correspondieron a representaciones sobre la pobreza, redes y mecanismos de apoyo social, sistemas de asociación y capital social, derechos ciudadanos, participación, y empoderamiento.

A través del análisis factorial, las variables con más grado de significación terminaron aportando a la construcción de tres factores significativos: a) el denominado dependencia de los programas de protección estatal que señala el grado de adhesión a los subsidios y programas públicos focalizados como estrategia contra la pobreza; b) la aceptación de la protección institucional que señala el grado de confianza y aprecio de las instituciones públicas (nacionales y distritales) que proveen programas y subsidios a los ciudadanos, en general, y a los pobres en particular, y c) el reconocimiento-cumplimiento, tanto de parte del Estado como de los ciudadanos, de un conjunto de deberes y derechos que caracterizan a un Estado social e indica el grado de aceptación de normas ciudadanas, como pagar impuestos y acatar las leyes, pero también el reconocimiento del grado en el que cumplen las autoridades con su función al proteger los derechos de los ciudadanos.

Con la técnica del cluster analysis se identificaron grupos que representaran conglomerados similares en su interior y distintos entre sí. Como se podrá observar más adelante, los cuatro grupos identificados se distribuyeron en un cuadrante que se obtiene con el método AFCM en dos direcciones: horizontalmente, de menos a más aceptación del tercer factor; verticalmente, de menos a más, a partir de la consideración de los dos primeros factores. Cada uno de estos grupos de bogotanos comparten opiniones, percepciones y representaciones similares, y tienen condiciones objetivas similares como gasto mensual en 
alimentos, estrato donde residen, nivel educativo, menores de edad presentes en el hogar, y haber o no nacido en la ciudad. Los grupos se denominaron beneficiarios confiados, empoderados, relativistas, y escépticos descontentos. Se consideró que estos grupos arrojan información figurada sobre los habitantes de Bogotá, a partir de su ubicación socioespacial y de sus representaciones sobre su particular condición social.

\section{Visión contextual y panorámica sobre la situación de pobreza en Colombia y Bogotá}

Desde la definición clásica de pobreza, Colombia se mantiene en una preocupante posición en el concierto latinoamericano, aunque con una tendencia a la disminución de las brechas. Es decir, se ha seguido reduciendo la pobreza por ingresos en el país, pero a un ritmo menor al de la media latinoamericana. Algunos datos fundamentan esta afirmación. Su tasa de pobreza ha pasado de 52.5\% en 1994, a 42.8\% en 2008 (CEPAL, 2009: 54) y 34\% en 2011 (Ángulo, Gaviria \& Morales, 2013). Además, el ingreso per cápita real creció 36\% entre 2002 y 2011 (Ángulo, Gaviria \& Morales, 2013). En este sentido, es posible afirmar que existe un lento progreso en la superación de la pobreza de Colombia frente a los países de la región de igual nivel. Sin embargo, variables como su largo y degradado conflicto armado interno, así como las dinámicas y lógicas económicas y políticas que lo mantienen activo (modelo de desarrollo desigual, concentración del ingreso, acaparamiento y despojo de tierras, desindustrialización y estilo productivo extractivista, entre otros) coadyuvan a su lentitud.

Examinado el tema con el índice de pobreza multidimensional, el descenso de la pobreza en Colombia ha sido más acelerado a partir de la década de 2010. De hecho, entre 2003 y 2010 el índice de pobreza multidimensional pasó del $49 \mathrm{al} \mathrm{30 \%} \mathrm{(Ángulo,} \mathrm{Gaviria} \mathrm{\&} \mathrm{Morales,} \mathrm{2013).} \mathrm{Desde} \mathrm{este} \mathrm{momento} \mathrm{el} \mathrm{proceso}$ continuó en disminución, en 2011 fue de 29.4\%, en 2012 de 27\% y en 2013 de $24.8 \%$. En términos de población por debajo de la línea de pobreza, esta fue de $40.3 \%$ en 2009 y de $30.6 \%$ en 2013 . Esta situación ha llevado a que lo ocurrido en el país entre 2002 y 2011 sea llamado la década ganada, especialmente para las clases medias que pasaron del 16 al 27\%; eso sí, con cifras más bajas en relación con Chile donde esta clase supera el 50\%, y México donde llega al 40\% (Ángulo, Gaviria \& Morales, 2013).

Para el caso de la ciudad de Bogotá, según cifras oficiales, el índice de pobreza multidimensional (IPM) también refleja una mejoría gradual en la situación respecto de otras regiones del país: $12.1 \%$ en $2010,11.9 \%$ en $2011,11.1 \%$ en 
2012, y 8.7\% en $2013 .{ }^{10}$ (Jiménez \& González, 2014). El porcentaje de población por debajo de la línea de pobreza, medido por ingresos en Bogotá, fue de $18.3 \%$ en 2009 y de $10.2 \%$ en 2013 . El índice de pobreza subjetiva en esta ciudad para 2013 fue de 16\% (Casa Editorial El Tiempo et al., 2014).

Una investigación como la que hemos propuesto sobre fronteras de la pobreza en Bogotá podría aportar a develar algunas de las múltiples dimensiones del tema en la capital y en otras urbes latinoamericanas, y funcionar como una referencia para la reflexión acerca de la ampliación de las definiciones convencionales, aportando también a las tradicionales políticas de intervención en este tema. A continuación, entraremos en detalle con lo arrojado en el proceso de pesquisa.

\section{Resultados y discusión analítica}

Este artículo considera que las fronteras de la pobreza en Bogotá dependen principalmente, aunque no en exclusiva, de tres factores explicativos resultado del análisis de correspondencias múltiples. Examinemos ahora cómo juegan estos factores en el espacio social figurado, a partir de los grupos identificados (gráficas 1 y 2). El análisis se realiza desde las variables activas que aportan en mayor y menor medida a cada uno de los factores y de las variables ilustrativas con más correlación en cada uno de los grupos.

$\mathrm{Al}$ indagar por las representaciones sobre la pobreza, asumimos como hipótesis que son condicionadas por el hecho de recibir o no subsidios y programas de alivio a la pobreza. Los programas de este tipo sobre los cuales se preguntó en 2008 fueron los servicios de salud a los cuales se accede a través del Sisbén, la educación pública, los comedores comunitarios, los programas de cuidado de menores y Familias en Acción. De todos estos programas, el que mayor frecuencia tuvo en las respuestas fueron los servicios de salud a través del Sisbén (42\%). Solo el $8 \%$ manifestó recibir o haber recibido los subsidios por Familias en Acción al momento del estudio.

\section{Los hallazgos en cada uno de los grupos identificados}

Las variables activas e ilustrativas que mayor peso tienen en la conformación de los cuatro grupos se presentan en los cuadros 1 y 2 en los anexos de este artículo. Al primer grupo lo denominamos beneficiarios confiados, pues, de los cuatro, es el más beneficiado por los programas y subsidios para aliviar la pobreza y el que,

10 El IPM se aplica para Bogotá desde el año 2003. 
en general, confía en que el gobierno y las autoridades distritales y nacionales lo protegen. Tiene el más bajo nivel educativo de los cuatro (44\% con primaria o menos), en su gran mayoría vive en estratos del uno al tres (97\%) y solo una tercera parte nació en Bogotá. Es el grupo que con mayor frecuencia declara que trabajar duro es condición para salir de la pobreza (66\%). Es optimista acerca de que el gobierno hace lo necesario para enfrentar la pobreza (65\% de acuerdo), aunque solo poco más de una décima parte recibe subsidios de Familias en Acción; es, de los cuatro, el grupo que en mayor medida los obtiene y seis de cada diez recibe servicios de salud a través del régimen subsidiado. Confía en que los gobiernos, tanto nacional como distrital, respetan sus derechos ciudadanos (77\%). Se caracteriza por emplear mecanismos que ayudan la asociatividad y a elevar su capital social. Para él son importantes los vecinos, parientes e iglesias, y después del segundo grupo, es el que tiene la mayor tasa de participación en un evento electoral en el último decenio (73\%). Su gasto mensual en alimentos es similar a dos de los cuatro grupos, pero es aquel con la mayor proporción de menores de edad en el hogar. Su uso de mecanismos para hacer valer sus derechos — como el derecho de petición y la acción de tutela - es el menor de los cuatro grupos y en 2008 , fecha de la encuesta, ningún miembro de este grupo usaba redes sociales como Facebook.

Gráfica 1. Plano social a partir de correspondencias múltiples con dos factores

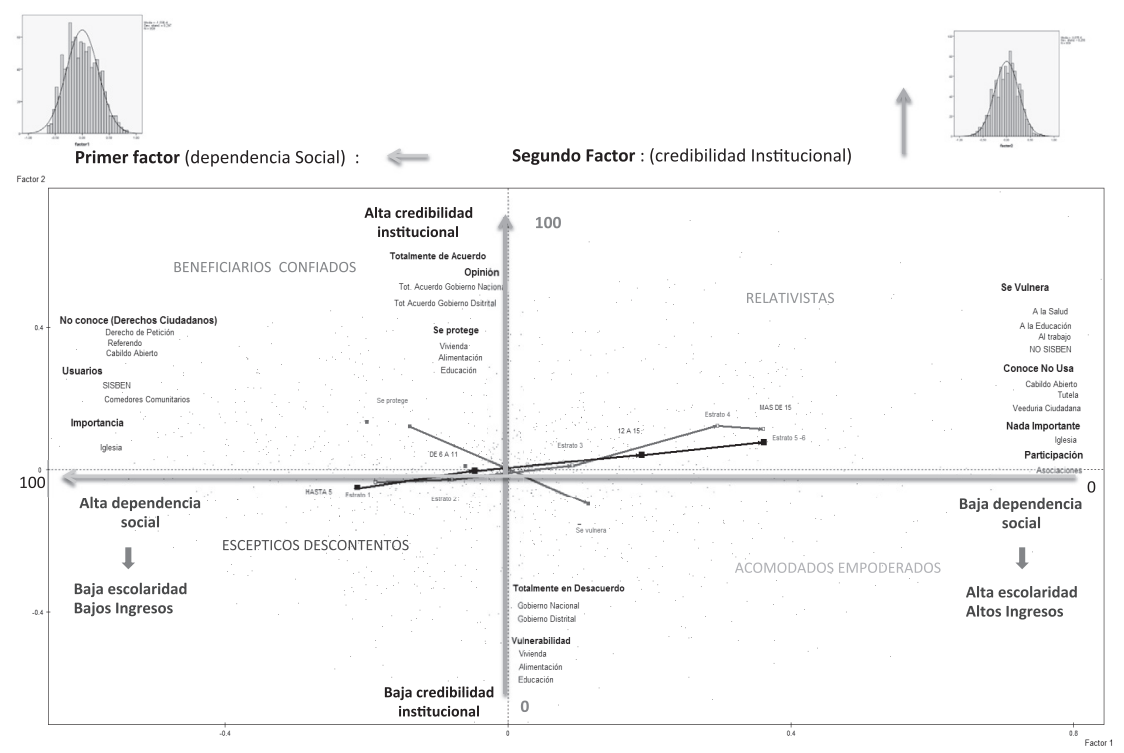

Fuente: Elaboración propia. 
Al segundo grupo lo denominamos empoderados. De los cuatro este es el grupo con mayor nivel educativo ( $42 \%$ con 15 años y más de educación), mayor gasto mensual en alimentos, menor proporción de menores de edad en el hogar (casi la mitad sin menores) y más propietarios de su residencia (64\%). De igual manera, es el de mayor participación en un evento electoral en el último decenio (89\%), el que más emplea las movilizaciones políticas para expresar su opinión, conoce y usa la acción de tutela, declara que pagar impuestos es una obligación ciudadana (92\%) y el que tiene mayor porcentaje de pertenencia a clubes, asociaciones de padres de familia y de egresados de colegios y de universidades. Es el grupo que reconoce en mayor medida que sus parientes son más importantes en su vida que sus vecinos (92\%). En el momento de la encuesta, era el grupo con mayor uso de redes sociales como Facebook (44\%), una práctica relacionada con su mayor nivel educativo y su nivel de ingresos. Es el grupo que con más frecuencia declara que las carencias afectivas y espirituales son más importantes que las materiales (82\%).

$\mathrm{Al}$ tercer grupo lo hemos denominado relativistas. Es el que contiene más proporción de jóvenes y de estudiantes, el segundo en proporción en declararse propietarios de casa propia (59\%), vive en estratos del uno al tres en porcentaje similar a los grupos uno y cuatro (90\%), pero es el segundo en residir en los estratos del cuatro al seis (10\%, contra 37\% para el grupo de empoderados). Es el que menos ha participado en eventos electorales, y quizá su relativa juventud explica en parte este comportamiento. Es el segundo en usar Facebook $(28 \%)$ y en su mayoría (64\%) se considera como clase media. Lo calificamos como relativista, porque en sus respuestas rara vez declara estar "totalmente de acuerdo" o "totalmente en desacuerdo" y, en cambio, tiende a dar respuestas que se acercan a la mitad de la escala.

Al cuarto grupo lo hemos denominado escépticos desconfiados. Aunque su residencia se ubica en estratos similares a los beneficiarios confiados y su gasto en alimentos, así como la proporción del grupo que declara ser propietaria de su casa es similar a dicho grupo, se diferencian de ellos y de los otros grupos por sus representaciones acerca "de no sentirse protegidos por el Estado". La mitad de los escépticos desconfiados ha recibido servicios de salud por el régimen subsidiado, pero tiene la menor proporción de personas que usan o conocen mecanismos de participación ciudadana (tutela, derecho de petición, movilizaciones), es el de menor porcentaje de membresía a iglesias y el que en menor proporción (65\%) declara que pagar impuestos es una obligación. También proporcionalmente es el grupo que menos declara (18\%) que el gobierno nacional o distrital hace lo necesario para enfrentar la pobreza o que vela por el cumplimiento de sus derechos ciudadanos (24\%, contra $77 \%$ en el primer grupo). 
Destaca en este breve panorama que tres de los cuatro grupos (el dos, el tres y el cuatro) declaran pertenecer a la clase media. En estos grupos, los empoderados tienen la mayor proporción (74\%) en este ítem, y el grupo de los escépticos representa un poco más de la mitad (54\%). La mayoría de los miembros de los cuatro grupos declaran haber estado en esa clase social toda la vida, mientras que la mayor movilidad social se declara por el grupo de empoderados, ya que solo el $68 \%$ indica haber estado en esa clase toda la vida, contra el $72 \%$ de los otros tres grupos.

En la gráfica 2 se visualiza la ubicación de las observaciones de cada uno de los cuatro grupos en un cuadrante marcado por los tres factores referenciados en el análisis.

Gráfica 2. Ubicación en el cuadrante de los cuatro grupos construidos

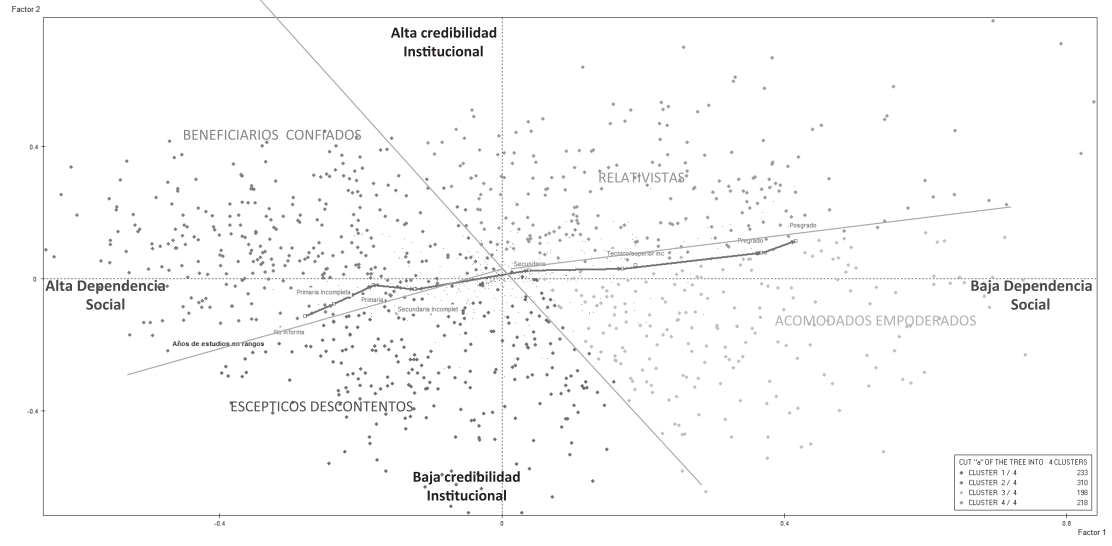

Fuente: Elaboración propia.

Discusión analítica

a) Corroborando lo afirmado en Uribe (2008), ${ }^{11}$ en una ciudad como Bogotá las fronteras de la pobreza se trazan a partir de ser o no sujetos de atención por vía de subsidios estatales. En el presente estudio, ello es claro en especial para el

11 Análisis triangulado de los resultados de dos encuestas aplicadas entre abril de 2005 (231 personas) y octubre de 2006 (1005 personas) y de 21 entrevistas personales realizadas en distintos estratos socioeconómicos. 
grupo uno, a través de programas de atención en salud del régimen subsidiado y, en menor medida, con los demás programas y subsidios para aliviar la pobreza.

b) En términos de factores explicativos, el análisis de los datos arroja que el factor uno (dependencia) es el que mayor varianza explica y es el que permite entender la diferencia más acentuada de fronteras de la pobreza entre los que reciben subsidios (beneficiarios confiados) y los que no los reciben (empoderados). El segundo factor (credibilidad) permite explicar las diferencias más ostensibles entre el grupo de los escépticos descontentos y los demás grupos, sobre todo porque los primeros perciben en sus representaciones que no son protegidos por el Estado nacional ni por la administración distrital. El tercer factor (cumplimiento-reconocimiento) permite entender que las diferencias más marcadas se encuentran entre los bogotanos con posiciones intermedias o en proceso de consolidación ("algo de acuerdo", "algo en desacuerdo"), en este caso los relativistas, en temas de representaciones sobre la pobreza y los bogotanos que tienen una posición consolidada en las mismas representaciones ("totalmente de acuerdo") y manifestando la importancia de instituciones como la familia, amigos o la Iglesia o de la necesidad de reconocimiento de la participación política (empoderados y beneficiarios confiados).

c) El bajo reconocimiento de los grupos uno y cuatro de los subsidios y de algunos programas de atención en la educación pública, los centros de cuidado de menores o los comedores comunitarios podría estar relacionado con inconvenientes de materialización de los programas en las vidas de estas personas en el distrito capital. Esto es evidente en los grupos focales con población en situación de desplazamiento en Bogotá, los cuales identificaron en su momento serios cuestionamientos a los programas y subsidios de vivienda y fueron enfáticos en señalar las innumerables dificultades administrativas enfrentadas para acceder a ciertos servicios.

d) Los residentes de la capital definieron quiénes deberían recibir subsidios y quiénes no. En general, en todos los grupos se piensa que los residentes de los estratos uno al tres deben recibir subsidios. Sin embargo, una proporción del grupo de empoderados también indica que nadie debería recibir subsidios. En cuanto al pago de impuestos, son marcadas las diferencias de opinión según el grupo al que se pertenece y depende de si actualmente se pagan impuestos o no.

e) El hecho de que todos los grupos tiendan a representarse en un lugar intermedio, como clase media, es una práctica de autoadscripción para ubicarse a la mitad de la escala social reportada en otros estudios y lugares (Climent, 2004). 
En este caso, se revela una traza de representación de la frontera de pobreza que se ubica en el estrato tres, pero el establecimiento de esta frontera varía en el rango, ubicándola entre los estratos dos y tres para los grupos de beneficiarios y el de los relativistas, y entre los estratos tres y cuatro para el grupo de empoderados. El hecho de que en Bogotá en el estrato tres viva el $40 \%$ de la población puede explicar que allí se ubique el punto medio de la distribución.

f) En las entrevistas y grupos focales el tener o no trabajo se constituye en frontera de sentido sobre la pobreza para tres de los grupos, mientras que para el grupo de los empoderados esta se ubica en tener o no suficientes ingresos o bienes. En la encuesta, la falta de trabajo es el primer elemento mencionado para ser considerado pobre en la ciudad de Bogotá (además de considerarse el derecho más violado, según tres de los grupos), aunque no se trata solamente de un empleo formal en el que media un contrato laboral, sino que se concibe como cualquier actividad que genere ingresos dignos y permanentes.

g) No poder dar educación a los hijos o atención médica cuando están enfermos es también un atributo decisivo para trazar las fronteras de la pobreza para la gran mayoría de los entrevistados en Bogotá. Esto coincide con lo encontrado en el estudio de Núnez \& Carvajal (2007) en Buenaventura y en el estudio de Voces de los pobres en Colombia (Arboleda, Petesch \& Blackburn, 2004: p. 82). Sin embargo, llama la atención cómo los grupos identifican que uno de los derechos que más se ha protegido a nivel distrital es el de la educación.

h) En las entrevistas y grupos focales tener casa propia es importante como traza para definir quién es o no es pobre. Es claro que trabajar para pagar un arriendo y no capitalizar esa suma en algún momento es un signo de vulnerabilidad. Pareciera que una vez que se adquiere vivienda o se tiene la seguridad de una casa propia, se cruza un umbral crítico hacia la no pobreza. Sin embargo, esto no coincide con lo hallado en las encuestas, pues solo entre el 36 y el 27\% de la muestra identifica que tener casa propia garantiza salir de la pobreza.

i) En las entrevistas y grupos focales se encontró un tema recurrente y es que, al evaluar la condición de pobreza, para los entrevistados siempre existía gente "más pobre" que ellos. En esta situación se hallarían las personas en situación de desplazamiento, las que viven en situación de indigencia, las que habitan en barrios invadidos y las que viven sin domicilio fijo.

j) Los grupos focales con población en situación de desplazamiento forzado permitieron entender la autopercepción de estos como personas marginales y 
marginadas en el orden social e institucional de la ciudad. Esta autocalificación está atravesada y condicionada por un imaginario de Bogotá como ciudad "impersonal", "poco solidaria", "devoradora" y "peligrosa".

k) Aunque el foco de atención estuvo en situar la condición de pobreza en aquellas categorías que invocaban con más fuerza el hecho de tener, por encima de aquellas que reflejaban el ser, las personas contactadas en el estudio también estuvieron "totalmente de acuerdo" en que las carencias afectivas y espirituales son más graves que las económicas. Sin embargo, a pesar de la percepción subjetiva tan marcada frente a la importancia de las carencias afectivas, también se percibe la crudeza objetiva de la pobreza como desprotección. Así, por ejemplo, para los grupos de beneficiarios confiados y escépticos descontentos se destaca una reiterada preocupación por su condición de inseguridad vital y su falta de control sobre el futuro y el entorno que los rodea.

l) Algunas de las personas entrevistadas y encuestadas sienten que no son solo pobres o se les percibe como pobres sino que tienen que vivir en barrios pobres e inseguros. Asimismo, señalan que los barrios en donde viven tienen problemas de seguridad. Tres de los grupos (los beneficiarios confiados, los empoderados y los escépticos descontentos) consideraron por encima del $60 \%$ que la seguridad depende del estrato donde se vive. En las entrevistas y grupos focales se observa que la vulnerabilidad de estos barrios incluye peligro en las calles, hurtos, consumo y venta de droga en parques y calles, circulación permanente de armas, reclutamiento de miembros de las familias por parte de grupos armados y delincuenciales y estrategias de revictimización. Esto coincide con resultados de algunos estudios sociológicos y psicológicos sobre los llamados efectos barriales, sobre la pobreza en otras partes del mundo (Harding, 2003; Oakes, 2004).

m) En todos los grupos se percibe que los pobres son más solidarios que los que no son pobres. Lo que sí varía por grupos, como se había comprobado en otros estudios, es que se piense, por parte del grupo de los beneficiarios confiados y el de los escépticos descontentos, que los residentes de los estratos más altos se aprovechan de los demás. Esta opinión contrasta con la expresada por el grupo de los empoderados, los que piensan que la condición de aprovecharse de los demás, si se da, es independiente del estrato en el que se vive, es decir, no está determinada por la estratificación de la ciudad.

n) Hacer uso de redes y asociaciones para salir adelante fue calificado como muy importante por la mayoría de los grupos. Sin embargo, como se indi- 
có, son los grupos de empoderados y relativistas los que más los emplean, y el grupo de los escépticos se destaca por el menor uso relativo de estas redes y de mecanismos de participación ciudadana. Es un hallazgo de este estudio que las diferencias "objetivas" entre los grupos uno y cuatro no son muy altas, pero en cambio su percepción sobre sentirse protegidos por el Estado, su uso de redes y asociatividad, y sus conceptos sobre sus obligaciones ciudadanas y políticas los colocan en orillas muy distintas.

\section{Reflexión concluyente}

En este artículo hemos identificado tres factores en particular que ayudan a comprender cómo las fronteras de la pobreza no se definen de una sola manera ni corresponden a criterios objetivos definidos por un déficit de ingresos o condiciones materiales de vida. Las políticas públicas, en especial aquellas destinadas a aliviar la pobreza, son determinantes en el delineamiento de estas fronteras. Ello ocurre, no solo mediante la identificación de la población que debe recibir el tratamiento antipobreza, por medio de mecanismos de focalización y selección de programas públicos y privados, sino también por el autorreconocimiento de parte de esa población de sentirse con derecho a recibirlo. Sin embargo, otros elementos influyen en esta ubicación en el espacio social figurado de los residentes, enmarcada en sus condiciones educativas, socioeconómicas y de ciertos capitales, entre los cuales se cuenta su nivel de participación política, su grado de asociatividad y su actitud respecto al esfuerzo personal necesario para salir de la pobreza.

\section{Referencias}

Alkire, S. (2007). The Missing Dimensions of Poverty Data: An Introduction. Oxford: University of Oxford-Department of International Development.

Alkire, S. \& Foster, J. (2009). Counting and Multidimensional Poverty Measurement. Oxford: University of Oxford-Poverty \& Human Development Initiative (орHI).

Angulo, R., Gaviria. A. \& Morales, L. (2013). La década ganada: evolución de la clase media y las condiciones de vida en Colombia, 2002-2011. Bogotá: Documentos del Centro de Estudios sobre Desarrollo Económico, Doc. 50.

Ángulo, R., Díaz, Y., Pardo, R. \& Riveros, Y. (2011), Índice de Pobreza Multidimensional para Colombia. Bogotá: Departamento Nacional de Planeación, Doc. 382. 
Arboleda, J., Petesch, P. \& Blackburn, J. (2004). Voces de los pobres de Colombia. Bogotá: Banco Mundial/Alfaomega.

Bayón, M. C. (2012). El "lugar" de los pobres: espacio, representaciones sociales y estigmas en la ciudad de México. Revista Mexicana de Sociología, 74(1), 133-166.

Bonilla, L. (2009). Determinantes de las diferencias regionales en la distribución del ingreso en Colombia, un ejercicio de microdescomposición. Ensayos sobre Política Económica, 27(59), 99-156.

Bourdieu, P. (1984). Distinction: A Social Critique of the Judgment of Taste. Londres: Routledge.

Casa Editorial El Tiempo (CEet), Fundación Corona, Cámara de Comercio de Bogotá, Pontificia Universidad Javeriana (2014). Programa Bogotá Cómo Vamos, Cómo Vamos en Localidades. Recuperado de: http://www.bogotacomovamos.org/documentos/informe-decalidad-de-vida-2014-3/

Ceccini, S. \& Madariaga, A. (2011). Conditional Cash Transfer Programmes. The Recent Experience in Latin America and the Caribbean. Santiago de Chile: Economic Commission for Latin America and the Caribbean (ECLAC)/Swedish International Development Cooperation Agency (Sida).

Comisión Económica para América Latina (2009). Panorama social de América Latina 2009. Santiago de Chile: CEPAL.

Climent, V. (2004). Estructura social de España y Cataluña. Barcelona: Universidad de Barcelona.

Escobar, A. (1996). La invención del Tercer Mundo. Construcción y deconstrucción del desarrollo. Bogotá: Norma.

Gollac, M. (2009). El rigor y la broma. Con respecto al uso de los métodos cuantitativos por Pierre Bourdieu. Sociogénesis. Revista Electrónica de Sociología. Recuperado de http://www. uv.mx/sociogenesis

Harding, D. (2003). Counterfactual Models of Neighborhood Effects: The Effect of Neighborhood Poverty on Dropping out and Teenage Pregnancy. American Journal of Sociology, 109(3), 676-719.

Jaramillo, J. (2008). Representaciones y prácticas discursivas sobre la política de atención a la población en situación de desplazamiento. Universitas Humanistica, 65(65), 197-234.

Jiménez, W. G. \& González, J. I. (2014). Calidad de vida urbana: una propuesta para su evaluación. Revista de Estudios Sociales, (49), 159-175. 
Jodelet, D. (1984). La representación social: fenómenos, conceptos y teoría. En Moscovici, S. (Ed.). Psicología social II. Pensamiento y vida social (pp. 469-494). Barcelona: Paidós.

Kakwani, N. \& Silber, S. (2008). Many Dimensions of Poverty. Nueva York: Palgrave Macmillan.

Lampis, A. (2011). Desafíos conceptuales para la política de protección social frente a la pobreza en Colombia. Revista de Estudios Sociales, 41,107-121.

Lebart, L., Morineau, A. \& Tabard, N. (1977). Techniques de la description statistique: méthodes et logiciels pour l'analyse des grands tableaux. París: Dunod-Bordas.

Liu, L. (2008). To Have and to Be: Towards the Social Representation of Quality of Life in China. Journal of Community \& Applied Social Psychology, 18(3), 233-252.

Moscovici, S. (2000). The History and Actuality of Social Representations. En Duveen, G. (Org.). Social Representations. Explorations in Social Psychology (pp. 120-155). Cambridge: Polity Press.

Narayan, D. (2000). La voz de los pobres ¿hay alguien que nos escuche? Madrid: Banco Mundial/ Mundiprensa.

Nuñez, J. \& Carvajal, A. (2007). Las voces de los pobres de Buenaventura ¿Cómo romper las trampas de pobreza? Bogotá: Cuadernos PNUD.

Oakes, M. (2004, mayo). The (Mis)Estimation of Neighborhood Effects: Causal Inference for a Practicable Social Epidemiology. Social Science \& Medicine, 58(10), 1929-1952.

Pardo, N. G. (2008). La representación de lo mensurable sobre la pobreza en la prensa colombiana. Discurso y Sociedad, 2(2), 394-421.

Pinzón, L. F. (2014). Los determinantes de la pobreza subjetiva en Colombia. Tesis de pregrado en Economía. Cali: Universidad del Valle.

Potter, J. (1998). La representación de la realidad. Discurso, retórica y construcción social. Barcelona: Paidós.

Programa de Naciones Unidas para el Informe de Desarrollo Humano para Bogotá. (2008). Bogotá: PNUD.

Ravallion, M. \& Lokshin, M. (1999). Subjective Economic Welfare. World Bank Policy Research Working Paper, (2106), 1-38. 
Roland-Levy, C., Kirchler, E., Penz, E. \& Gray, C. (Eds.). (2001). Everyday Representations of the Economy. Viena: Universitatsverlag.

Rojas, C. (2004). Citizenship and the Social Question in Colombia. Paper Presented at the Annual Meeting of the International Studies Association, Le Centre Sheraton Hotel, Montreal, Quebec, Canadá.

Rojas, M. \& Jiménez, E. (2008, julio-diciembre). Pobreza subjetiva en México: el papel de las normas de evaluación del ingreso, Perfiles Latinoamericanos, (32), 11-33.

Sen, A. (1976). Un enfoque ordinal para medir la pobreza. Cuadernos de Economía, XVII (29), 39-65.

Spicker, P., Álvarez, S. \& Gordon, D. (2009). Pobreza: Un glosario internacional. Buenos Aires: Consejo Latinoamericano de Ciencias Sociales.

Uribe, C. (2008). Estratificación social en Bogotá: de la política pública a la dinámica de la segregación social. Universitas Humanistica, 65 (65), 39-171.

Uribe, C., Vásquez, S. \& Pardo, C. (2006). Subsidiar y segregar: la política de estratificación y sus efectos sobre la movilidad social en Bogotá. Papel Político, 11(1), 69-93.

Vasilachis, I. (2003). Pobres, pobreza, identidad y representaciones sociales. Barcelona: Gedisa. 


\section{Anexos}

Cuadro 1. Variables activas con mayor peso en los tres factores identificados (en \%)

\begin{tabular}{|c|c|c|c|c|c|}
\hline & & Beneficiarios & Empoderados & Relativistas & Escépticos \\
\hline \multirow{12}{*}{$\begin{array}{l}\text { Representaciones } \\
\text { sobre la pobreza }\end{array}$} & $\begin{array}{l}\text { Trabajar duro es condición para } \\
\text { no ser pobre }\end{array}$ & 66 & 39 & 59 & 52 \\
\hline & $\begin{array}{l}\text { Los de estratos altos se aprove- } \\
\text { chan de los demás }\end{array}$ & 74 & 42 & 59 & 74 \\
\hline & $\begin{array}{l}\text { Existe más solidaridad entre } \\
\text { vecinos de estratos inferiores que } \\
\text { de estratos superiores }\end{array}$ & 82 & 74 & 78 & 77 \\
\hline & $\begin{array}{l}\text { Las carencias afectivas y espiri- } \\
\text { tuales son más importantes que } \\
\text { las económicas }\end{array}$ & 74 & 82 & 73 & 71 \\
\hline & $\begin{array}{l}\text { La pobreza está asociada con } \\
\text { una falta de derechos }\end{array}$ & 76 & 61 & 70 & 83 \\
\hline & $\begin{array}{l}\text { Cuando se tiene casa propia se } \\
\text { deja de ser pobre }\end{array}$ & 36 & 27 & 38 & 26 \\
\hline & $\begin{array}{l}\text { Tener derechos en Colombia es } \\
\text { un asunto de privilegios }\end{array}$ & 65 & 56 & 57 & 76 \\
\hline & Se considera de clase media & 48 & 74 & 64 & 54 \\
\hline & $\begin{array}{l}\text { Ha estado en esa clase toda } \\
\text { la vida }\end{array}$ & 78 & 68 & 72 & 77 \\
\hline & $\begin{array}{l}\text { El gobierno hace lo necesario } \\
\text { para enfrentar la pobreza }\end{array}$ & 65 & 29 & 35 & 18 \\
\hline & $\begin{array}{l}\text { Los gobiernos nacional y distrital } \\
\text { velan por el cumplimiento de sus } \\
\text { derechos ciudadanos }\end{array}$ & 77 & 48 & 60 & 24 \\
\hline & $\begin{array}{l}\text { El pago de impuestos es una } \\
\text { obligación }\end{array}$ & 80 & 92 & 79 & 65 \\
\hline \multirow{8}{*}{$\begin{array}{l}\text { Redes, asociacio- } \\
\text { nes, capital social, } \\
\text { conocimiento y } \\
\text { uso de derechos }\end{array}$} & $\begin{array}{l}\text { Desconoce el mecanismo de } \\
\text { acción de tutela }\end{array}$ & 59 & 4 & 38 & 49 \\
\hline & $\begin{array}{l}\text { Ha ido a marchas callejeras o } \\
\text { movilizaciones }\end{array}$ & 19 & 44 & 40 & 15 \\
\hline & $\begin{array}{l}\text { Ha participado en algún evento } \\
\text { electoral en los últimos diez años }\end{array}$ & 73 & 89 & 59 & 63 \\
\hline & $\begin{array}{l}\text { Los parientes son importantes } \\
\text { en su vida }\end{array}$ & 80 & 92 & 79 & 65 \\
\hline & Miembro activo de una Iglesia & 51 & 42 & 37 & 31 \\
\hline & $\begin{array}{l}\text { Recibe o ha recibido subsidios } \\
\text { de Familias en Acción }\end{array}$ & 13 & 2 & 5 & 9 \\
\hline & $\begin{array}{l}\text { Beneficiario de servicios de salud } \\
\text { por régimen subsidiado }\end{array}$ & 60 & 13 & 35 & 50 \\
\hline & Usa Facebook & 0 & 44 & 28 & 5 \\
\hline \multicolumn{2}{|c|}{ Número de personas en cada categoría } & 294 & 179 & 229 & 257 \\
\hline
\end{tabular}

Fuente: Encuesta del estudio, 2008. 
Cuadro 2. Variables ilustrativas con mayor correlación para los cuatro grupos (en \%)

\begin{tabular}{lcccc}
\hline & Beneficiarios & Empoderados & Relativistas & Escépticos \\
\hline Viven en estratos uno al tres & 97 & 63 & 90 & 95 \\
Viven en estratos cuatro al seis & 3 & 37 & 10 & 5 \\
Mayores de 55 años & 37 & 23 & 12 & 25 \\
Amas de casa & 24 & 8 & 15 & 21 \\
Estudiantes & 6 & 15 & 22 & 7 \\
Empleados & 22 & 30 & 27 & 29 \\
Gasto mensual per cápita en alimentos & 35 & 61 & 35 & 36 \\
50 usD y más & 32 & 55 & 60 & 47 \\
Nació en Bogotá & 63 & 58 & 39 & 59 \\
Jefes de hogar & 2 & 42 & 9 & 6 \\
Nivel educativo 15 años y más & 29 & 47 & 34 & 32 \\
Sin menores de edad en casa & 52 & 64 & 59 & 51 \\
Casa propia & 21 & 13 & 13 & 12 \\
Ningún residente debe recibir subsidios del & 294 & 179 & 229 & 257 \\
Estado & & & & \\
\hline Número de personas en cada categoría &
\end{tabular}

Fuente: Encuesta del estudio, 2008.

Recibido el 15 de diciembre de 2014.

Aceptado el 21 de agosto de 2015. 